\title{
EXISTENCE OF SMOOTH SOLUTIONS TO THE CLASSICAL MOMENT PROBLEMS
}

\author{
PALLE E. T. JORGENSEN
}

\begin{abstract}
Let $s(0), s(1), \ldots$ be a given sequence, and define $s(n)=\overline{s(-n)}$ for $n<0$. If $\sum \sum \bar{\xi}_{n} \xi_{m} s(m-n) \geq 0$ holds for all finite sequences $\left(\xi_{n}\right)_{n \in \mathbf{Z}}$, then it is known that there is a positive Borel measure $\mu$ on the circle $\mathbb{T}$ such that $s(n)=\int_{-\pi}^{\pi} e^{i n t} d \mu(t)$, and conversely. Our main theorem provides a necessary and sufficient condition on the sequence $(s(n))$ that the measure $\mu$ may be chosen to be smooth. A measure $\mu$ is said to be smooth if it has the same spectral type as the operator $i d / d t$ acting on $L^{2}(\mathbb{T})$ with respect to Haar measure $d t$ on $\mathbb{T}$ : Equivalently, $\mu$ is a superposition (possibly infinite) of measures of the form $|w(t)|^{2} d t$ with $w \in L^{2}(\mathbb{T})$ such that $d w / d t \in L^{2}(\mathbb{T})$. The condition is stated purely in terms of the initially given sequence $(s(n))$ : We show that a smooth representation exists if and only if, for some $\varepsilon \in \mathbb{R}_{+}$, the $a$ priori estimate

$$
\sum \sum s(m-n) \bar{\xi}_{n} \xi_{m} \geq \varepsilon\left|\sum n s(n) \xi_{n}\right|^{2}
$$

is valid for all finite double sequences $\left(\xi_{n}\right)$. An analogous result is proved for the determinate (Hamburger) moment problem on the line. But the corresponding result does not hold for the indeterminate moment problem.
\end{abstract}

\section{INTRODUCTION}

Given an infinite sequence of numbers $s(n), n \in \mathbb{Z}$, the trigonometric moment problem [Ak] concerns (the familiar) conditions on the sequence which allow a representation

$$
s(n)=\int_{-\pi}^{\pi} e^{i n t} d \mu(t), \quad n \in \mathbb{Z},
$$

for some positive Borel measure $\mu$ on the circle. The corresponding problem for the real line (solved first by Hamburger [Ha]) asks, for a given sequence $s(0), s(1), \ldots$, for a (finite) positive Borel measure $\nu$ on $\mathbb{R}$ such that

$$
s(n)=\int_{\mathbb{R}} x^{n} d \nu(x), \quad n=0,1,2, \ldots
$$

In this note, we shall use harmonic analysis and operator theory to solve the restricted problem (for the circle $\mathbb{T}^{1}$ and the line $\mathbb{R}$ ) which asks for a

Received by the editors March 8, 1990 and, in revised form, June 18, 1990.

1980 Mathematics Subject Classification (1985 Revision). Primary 46H15, 46K10, 42A70, 42A05; Secondary $28 \mathrm{C} 15,42 \mathrm{~A} 82,43 \mathrm{~A} 35$.

Work supported in part by the NSF. 
representation of a given sequence in terms of a "smooth" measure. Both of the groups $\mathbb{T}^{1}$ and $\mathbb{R}$ have the respective Haar measures, and we ask for the representing measures to be absolutely continuous with respect to Haar measure, and to satisfy an additional smoothness condition.

Definition 1.1. Let $G$ be a locally compact abelian group, and let $d t$ denote the (unique up to scalar multiple) Haar measure on $G$. Let $L^{2}(G)$ be the Hilbert space of all square integrable functions on $G$ (with respect to Haar measure). We say that a measure $\mu$ on $G$ is smooth if it is of the form $|w(t)|^{2} d t$ for some $w \in L^{2}(G)$ with $L^{2}$-derivative, or if it is a superposition of such measures, i.e., there is a sequence $w_{k} \in L^{2}(G), k=1,2, \ldots$, each $w_{k}$ with $L^{2}$-derivative, such that $\sum_{k}\left|w_{k}\right|^{2} \in L^{1}(G), \sum_{k}\left|w_{k}^{\prime}\right|^{2} \in L^{1}(G)$, and the measure $\mu$ is represented as a sum $\sum_{k}\left|w_{k}(t)\right|^{2} d t$.

Such representations arise frequently in spectral theory for operators [Jo] which, in turn, are studied in terms of moment theory. The representation holds on the absolutely continuous part of the spectrum of the operator under consideration.

\section{THE MAIN THEOREM}

We shall give necessary and sufficient conditions (applicable to the two moment problems) which are stated directly in terms of the given sequence. The sequence will have a representation, (1.1) resp., (1.2), with a smooth measure if and only if the respective conditions are satisfied.

We begin with the trigonometric moment problem where the result is easier to state because representing measures are automatically unique, see e.g., [B-C] or [La1].

Theorem 2.1. Let $s(0), s(1), s(2), \ldots$ be a scalar sequence, and define, for $n<$ $0, s(n):=\overline{s(-n)}$. Then there is a smooth positive Borel measure $\mu$ on the circle such that

$$
s(n)=\int_{-\pi}^{\pi} e^{i n t} d \mu(t)
$$

if and only if there is an $\varepsilon \in \mathbb{R}_{+}$with the property that the a priori estimate

$$
\sum_{n} \sum_{m} \bar{\xi}_{n} \xi_{m} s(m-n) \geq \varepsilon\left|\sum_{n} n s(n) \xi_{n}\right|^{2}
$$

is satisfied for all complex (finite) sequences $\left(\xi_{n}\right)_{n \in \mathbb{Z}}$.

In the remark below, we first sketch why (2.2) is necessary. The proof of sufficiency represents the main portion of the paper. The technical lemmas are included in $\S 3$.

Remark 2.2. The condition (2.2) is clearly necessary. If a smooth $\mu$ exists as specified, pick functions $w_{k} \in L^{2}(d t)$ (where $d t$ denotes Haar measure on the circle) such that $d \mu(t)=\sum\left|w_{k}(t)\right|^{2} d t$, and $w_{k}^{\prime}=d w_{k} / d t \in L^{2}(d t)$ again with integrable square sum. When $p$ is given by a finite Laurent expansion, 
$p\left(e^{i t}\right)=\sum_{n} \xi_{n} e^{i n t}$, then

$$
\begin{aligned}
\left|\sum n s(n) \xi_{n}\right| & =\left|\int_{\mathbb{T}^{1}} p^{\prime} d \mu\right|=\left|\sum \int p^{\prime} w_{k} \bar{w}_{k} d t\right| \\
& =\left|\sum \int p\left(w_{k}^{\prime} \bar{w}_{k}+w_{k} \bar{w}_{k}^{\prime}\right) d t\right| \\
& \leq 2\left(\sum \int\left|w_{k}^{\prime}\right|^{2} d t\right)^{1 / 2}\left(\sum \int|p|^{2}\left|w_{k}\right|^{2} d t\right)^{1 / 2} \\
& =C\left(\int|p|^{2} d \mu\right)^{1 / 2}=C\left(\sum \sum \bar{\xi}_{n} \xi_{m} s(m-n)\right)^{1 / 2}
\end{aligned}
$$

which is the desired estimate for $\varepsilon=C^{-2}$.

The proof that (2.2) is also sufficient for existence of a smooth representation is nontrivial.

\section{DetaIls OF PROOF (SUFFICIENCY OF (2.2))}

We first review the construction of the measure $\mu$. The discussion is couched in terms of algebras and representations. These will be needed in $\S \S 4-5$ below.

Consider the Hilbert space $\mathscr{H}$ obtained by an application of the GelfandNaimark-Segal construction [Po] to the quadratic form $\sum_{n} \sum_{m} s(n-m) \xi_{n} \bar{\xi}_{m}$ which is certainly positive semidefinite by virtue of assumption (2.2) above. Recall that we have extended the given sequence $s(0), s(1), s(2), \ldots$ to be defined also for $n<0$ by setting $s(n)=\overline{s(-n)}$. Let $p(z)=\sum_{n} \xi_{n} z^{n}$ be a trigonometric polynomial (i.e., $\exists N>0$ such that the coefficient sequence $\left(\xi_{n}\right)$ satisfies, $\xi_{n}=0$ for all $\left.n,|n|>N\right)$, and let $\mathscr{P}$ denote the vector space of all such trigonometric polynomials defined on $\mathbb{T}^{1}$.

Then the Hilbert space $\mathscr{H}$ is obtained as a completion of this space $\mathscr{P}$ of all trigonometric polynomials, quotient out by the radical consisting of $p(\cdot)$ satisfying

$$
\sum_{n} \sum_{m} s(n-m) \xi_{n} \bar{\xi}_{m}=0
$$

It follows from the Cauchy-Schwarz inequality that both of trigonometric polynomials $z p(z)$ and $\bar{z} p(z)$ (defined on $\mathbb{T}^{1}$ where $\bar{z}=z^{-1}$ ) satisfy (3.1) whenever $p(\cdot)$ does. Since the trigonometric polynomials are dense in $C\left(\mathbb{T}^{1}\right)$ by the Stone-Weierstrass Theorem, it follows that a unitary operator $V$ on $\mathscr{H}$ is defined by

$$
(V p)(z)=z p(z), \quad z \in \mathbb{T}^{1}, p \in \mathscr{P} .
$$

Let, $p \rightarrow[p]$, denote the quotient mapping, embedding $\mathscr{P}$ as a dense linear subspace of $\mathscr{H}$, and let 1 denote the constant one element in $\mathscr{P}$. Then the vector [1] is cyclic in $\mathscr{H}$ for the operator group $\left\{V^{n}: n \in \mathbb{Z}\right\}$, and

$$
s(n)=\left\langle[1], V^{n}[1]\right\rangle
$$

where $\langle\cdot, \cdot\rangle$ denotes the inner product of the above mentioned Hilbert space $\mathscr{H}$. For $p(z)=\sum \xi_{n} z^{n}$, and $q(z)=\sum \eta_{n} z^{n}$ given elements in $\mathscr{P}$, we have $\langle p, q\rangle=\sum_{n} \sum_{m} \bar{\xi}_{m} \eta_{n} s(n-m)$. The validity of formula (3.3) is part of the conclusion of the GNS-construction. Also note that (3.3) provides a solution 
(the solution, in fact!) to the moment problem for the given sequence $\{s(n)\}$. For, if $P(\cdot)$ denotes the spectral resolution of $V$, then the formula,

$$
\mu(\cdot):=\langle[1], P(\cdot)[1]\rangle
$$

defines a positive Borel measure on the circle group $\mathbb{T}^{1}$ (which is now viewed as the dual compact Abelian group for $\mathbb{Z}$ ). This follows immediately from substitution of the expression

$$
V^{n}=\int_{\mathbb{T}^{1}} z^{n} P(d z), \quad n \in \mathbb{Z},
$$

into (3.3) above, and using that $P(\cdot)$ is an orthogonal projection-valued Borel measure on. $\mathbb{T}^{1}$.

Let $f$ be a bounded Borel function on $\mathbb{T}^{1}$, and let $f(V)$ be the corresponding operator defined by the functional calculus

$$
f(V)=\int_{\mathbb{T}^{1}} f(z) P(d z) .
$$

Then the assignment

$$
f(V)[1] \mapsto f(z)
$$

defines a unitary isomorphism of $\mathscr{H}$ onto $L^{2}\left(\mathbb{T}^{1}, d \mu\right)$. This follows again from a combination of formula (3.3) above, the spectral theorem, and the StoneWeierstrass Approximation Theorem. Under this mapping, the vector [1] in $\mathscr{H}$ is mapped onto the constant function in $L^{2}(\mu)$. When the measure $\mu$ is normalized, corresponding to taking $s(0)=1$, we get [1] mapped to the constant function one in $L^{2}(\mu)$.

The following lemma is based on the argument from [B-R, Proposition 3.2.28]. Having the above-mentioned isomorphism, we may identify $\mathscr{P}$ with a dense linear subspace of $L^{2}(\mu)$ where $\mu$ is the representing measure for $(s(n))$, which we take to be a probability measure on $\mathbb{T}^{1}$. If $p(\cdot)$ is given by the finite Laurent series, $p(z)=\sum_{n} \xi_{n} z^{n}$, then $p(V)=\sum \xi_{n} V^{n}$. Since $V$ is unitary, we have, for $n<0, V^{n}=\left(V^{*}\right)^{-n}$. We further define the derivative $p^{\prime}$ by the Laurent series

$$
p^{\prime}(z)=\sum_{n} n \xi_{n} z^{n}
$$

With this terminology, we have

Lemma 3.1. There is a symmetric operator $D$ in $L^{2}(\mu)$ and a vector $\psi \in L^{2}(\mu)$ such that $\mathscr{P}$ is contained in the domain of $D$, and further

$$
D p=p^{\prime}+p(V) \psi, \quad p \in \mathscr{P} \text {. }
$$

Proof. Let $p \in \mathscr{P}$ be given with Laurent series $\xi_{n}$, and let $p^{*}$ be the trigonometric polynomial with Laurent series, $n \mapsto \bar{\xi}_{-n}$. Then it follows that

$$
\left(p^{\prime}\right)^{*}=-\left(p^{*}\right)^{\prime} ;
$$

and further that

$$
(p q)^{\prime}=p^{\prime} q+p q^{\prime}
$$

for all $p, q \in \mathscr{P}$. 
The estimate (2.2), may be rewritten in the form,

$$
\left|\sum_{n} n \xi_{n} s(n)\right| \leq C\|p\|_{L^{2}(\mu)},
$$

now valid for some fixed (finite) constant $C$. From the Riesz-representation theorem, we get some $\psi_{0} \in L^{2}(\mu)$ satisfying

$$
\sum_{n} n \xi_{n} s(n)=\left\langle\psi_{0}, p\right\rangle=\int_{\mathbb{T}^{1}} \bar{\psi}_{0} p d \mu .
$$

Using this identity, and (3.9), we then conclude that $\bar{\psi}_{0}+\psi_{0}=0$, i.e. that $\psi_{0}$ takes purely imaginary values almost everywhere (w.r.t. $\mu$ ) on $\mathbb{T}^{1}$. Now apply identity (3.10) to $p^{*} q$ for an arbitrary pair of elements $p, q$ in $\mathscr{P}$. When the property of $\psi_{0}$ is used, we conclude that the operator $D$ in $L^{2}(\mu)$, defined by $D p=p^{\prime}+\frac{1}{2} p(V) \psi_{0}$, is symmetric, i.e., $\langle D p, q\rangle=\langle p, D q\rangle$ where $\langle p, D q\rangle=\int_{\mathbb{T}^{1}} \bar{p}(D q) d \mu$. It follows that the function $\psi:=\frac{1}{2} \psi_{0}$ satisfies the properties stated in the lemma.

We shall also need the

Lemma 3.2. The operator pair $D, V$ in $L^{2}(\mu)$ satisfies the commutation relation

$$
V^{n} D\left(V^{n}\right)^{*}=D-n I
$$

for all $n \in \mathbb{Z}$, as an operator identity on $\mathscr{P}$ where $I$ denotes the identity operator.

Proof. The conclusion of the lemma is a direct consequence of formulas (3.8) and (3.10) above. Note that, in the function representation (3.6) of $\mathscr{H}$ as $L^{2}(\mu)$, the operator $p(V)$ in (3.8) is multiplication by the function, $z \rightarrow p(z)$ on $\mathbb{T}^{1}$, and this is a bounded operator since multiplication by $z$ (on $\mathbb{T}^{1}$ ) is unitary on $L^{2}(\mu)$. However, $\psi$ may be unbounded.

\section{EXTENDING THE REPRESENTATION}

Our next step is based on an extension theorem for operator systems of the form (3.12). The operator $D$ in (3.8) is symmetric but generally not selfadjoint. The corresponding deficiency-indices $[A-G]$ measure the lack of selfadjointness. Recall that these indices are defined as the co-dimensions of the respective linear subspaces, $(D \pm i I) \mathscr{P}$ in $L^{2}(\mu)$ where $i=\sqrt{-1}$. The theorem states that it is possible to make the operator $D$ selfadjoint provided we pass to a bigger Hilbert space $\widetilde{\mathscr{H}}$ containing the given one $L^{2}(\mu)$, and the commutation relation $(3.12)$ may be "lifted" to $\widetilde{\mathscr{H}}$. See [J-Mo] for more details on commutation relations.

This theorem was proved first in [J-Mu] for the special case when at least one of the two deficiency-indices is assumed to be finite; and the finiteness restriction was then removed by Werner in the recent paper [We].

However, the application in [Jo2, J-Mu and We] are for Weyl systems on the real line. The present discussion will be based on the group duality $\mathbb{Z}-\mathbb{T}$, and the present arguments are somewhat different.

By general theory $[\mathrm{A}-\mathrm{G}]$, we may pick some Hilbert space $\mathscr{K}$, containing $\mathscr{H}$ as a closed subspace, and a selfadjoint operator $K$ in $\mathscr{K}$ such that

$$
D p=K p \text { for all } p \in \mathscr{P}
$$


where we recall that $\mathscr{P}$ is viewed as a dense linear subspace of $\mathscr{H}=L^{2}(\mu)$. Let $E(\cdot)$ be the spectral resolution of $K$, i.e., the orthogonal projection valued measure on $\mathbb{R}$, taking values in the orthogonal projections of $\mathscr{K}$, and satisfying, $K=\int_{\mathbb{R}} \lambda E(d \lambda)$. Let $Q$ be the orthogonal projection of $\mathscr{K}$ onto $\mathscr{H}$, and set

$$
F(\cdot)=Q E(\cdot) Q
$$

We then get

$$
D p=\int_{\mathbb{R}} \lambda F(d \lambda) p, \quad p \in \mathscr{P} .
$$

Let $\mathscr{F}$ be the set of all such (generally nonorthogonal) positive spectral resolutions. We shall need the following action of $\mathbb{Z}$ on $\mathscr{F}$. Let $\alpha_{n}$ be given by $(n \in \mathbb{Z})$,

$$
\alpha_{n}(F)(\lambda)=F(\lambda+n), \quad F \in \mathscr{F},
$$

and

$$
\beta_{n}(F)=V^{-n} \alpha_{n}(F) V^{n} .
$$

Then $\beta_{n}(F) \in \mathscr{F}$, whenever $F \in \mathscr{F}$ and $n \in \mathbb{Z}$. This follows from Lemma 3.2 above.

Using now amenability of $\mathbb{Z}$, weak ${ }^{*}$-compactness of $\mathscr{F}$, and the MarkovKakutani-fixed point theorem [D-S], we get some $F_{0} \in \mathscr{F}$ satisfying $\beta_{n}\left(F_{0}\right)=$ $F_{0}, n \in \mathbb{Z}$. This may be rewritten in the form

$$
V^{n} F_{0}(d \lambda) V^{-n}=F_{0}(d \lambda+n), \quad n \in \mathbb{Z} .
$$

Alternatively, define $F_{0}(\varphi):=\int_{\mathbb{R}} \varphi(\lambda) F_{0}(d \lambda)$ for compactly supported continuous functions $\varphi$ on $\mathbb{R}$. Then the commutation relation takes the form

$$
V^{n} F_{0}(\varphi) V^{-n}=F_{0}\left(\varphi_{n}\right)
$$

where the notation $\varphi_{n}$ refers to the translation action of $\mathbb{Z}$ on $\varphi$, see (4.7) below.

Using the extension method from [ $\mathrm{J}-\mathrm{Mu}, \S 7]$, we may choose an isometric embedding, $W: \mathscr{H} \rightarrow \widetilde{\mathscr{H}}$, and an orthogonal projection measure $E_{0}$ in $\widetilde{\mathscr{H}}$ such that $F_{0}(\cdot)=W^{*} E_{0}(\cdot) W$; and $V$ may be extended to a unitary operator $\widetilde{V}$ in $\widetilde{\mathscr{H}}$ such that

$$
\tilde{V}^{n} E_{0}(\varphi)(\tilde{V})^{-n}=E_{0}\left(\varphi_{n}\right)
$$

where again $\varphi_{n}$ denotes the translation,

$$
\varphi_{n}(\lambda):=\varphi(\lambda-n), \quad \lambda \in \mathbb{R}, n \in \mathbb{Z} .
$$

The measure $E_{0}(\cdot)$ may possibly be continuous; but, in any case, a Borel subset $\Delta \subset \mathbb{R}$ may be chosen such that the projections $\left(E_{0}(\Delta+n)\right)_{n \in \mathbb{Z}}$ are nonzero and mutually orthogonal. From the construction of $(\widetilde{V}, \widetilde{\mathscr{H}})$ in $[\mathrm{J}-\mathrm{Mu}]$, it follows further that $\mathscr{H}$ will be a reducing subspace for $\widetilde{V}$, acting on the (dilated) space $\widetilde{\mathscr{H}}$. Let, for $m \in \mathbb{Z}, \widetilde{E}_{m}:=E_{0}(\Delta+m)$. Then

$$
\widetilde{V}^{n} \widetilde{E}_{m}(\widetilde{V})^{-n}=\widetilde{E}_{m+n}
$$

for all pairs $n, m \in \mathbb{Z}$, which is the desired commutation relation. When the Stone-von Neumann uniqueness theorem [Jo2, Chapter 4] is applied, we get the 
system $\left(\tilde{V}, \widetilde{E}_{m}\right)$ concretely realized on the Hilbert space $L^{2}\left(\mathbb{T}^{1}, d t, \mathscr{M}\right)$ of $L^{2}$-vector valued functions on the circle, with values in some Hilbert space $\mathscr{M}$ (depending on the multiplicity). The measure $d t$ on $\mathbb{T}^{1}$ refers to Haar measure. On $f: \mathbb{T}^{1} \rightarrow \mathscr{M}, f \in L^{2}\left(\mathbb{T}^{1}, d t, \mathscr{M}\right)$, we have $\|f\|^{2}=\int\left\|f\left(e^{i t}\right)\right\|_{\mathscr{M}}^{2} d t$, and the operator $\tilde{V}$ is realized as

$$
(\tilde{V} f)(z)=z f(z), \quad z \in \mathbb{T}^{1} .
$$

Let $W: \mathscr{H} \rightarrow L^{2}(\mathbb{T}, d t, \mathscr{M})$ denote the isometric embedding which results from the application of the uniqueness theorem. Then, for $n \in \mathbb{Z}$, we have

$$
s(n)=\int_{\mathbb{T}^{1}} z^{n} d \mu=\left\langle[1], V^{n}[1]\right\rangle=\left\langle W[1], \widetilde{V}^{n} W[1]\right\rangle
$$

where the last inner product is in $L^{2}\left(\mathbb{T}^{1}, d t, \mathscr{M}\right)$. Let $w=W[1]$. Then

$$
s(n)=\int e^{i n t}\left\|w\left(e^{i t}\right)\right\|_{\mathscr{M}}^{2} d t
$$

If $w(\cdot)$ is expanded relative to some orthonormal basis for $\mathscr{M}$, we get the desired representation for the representing measure $\mu$. Let $w_{k}(\cdot)$ be the coordinate functions relative to the chosen ONB in $\mathscr{M}$. Then the $w_{k}$ 's are scalar functions, and

$$
\left\|w\left(e^{i t}\right)\right\|_{\mathscr{M}}^{2}=\sum_{k}\left|w_{k}\left(e^{i t}\right)\right|^{2} .
$$

It follows from (4.10) and (2.1) that

$$
d \mu(t)=\sum_{k}\left|w_{k}\left(e^{i t}\right)\right|^{2} d t
$$

is a representation as specified in Definition 1.1 above.

We also have the smoothness condition satisfied. This follows from the representation of the operator group

$$
T(\zeta)=\sum_{m \in \mathbb{Z}} \zeta^{m} \widetilde{E}_{m}, \quad \zeta \in \mathbb{T},
$$

on $L^{2}(\mathbb{T}, d t, \mathscr{M})$ in the form,

$$
T(\zeta) f(z)=f(\zeta z), \quad z, \zeta \in \mathbb{T}
$$

(which is translation on the circle in terms of the angular variable).

Formula (4.8) then takes the form

$$
\widetilde{V}^{n} T(\zeta)(\tilde{V})^{-n}=\zeta^{-n} T(\zeta)
$$

and the smoothness property of the vector function $w=W[1]$ follows from having $w$ in the domain of the infinitesimal generator of the unitary group $T(\cdot)$ given in (4.12). This generator is an extension (by construction, see (4.2)) of the operator $D$ from Lemma 3.1, and the vector [1] is in the domain of $D$. This is part of the conclusion of Lemma 3.1 .

\section{The HAMBURger MOMENT PROBlem}

Smoothness conditions for the Hamburger moment problem (1.2), for the line, are more complicated than the condition (2.2) from Theorem 2.1 regarding the trigonometric moment problem. This is due, in part, to the fact [Ak] 
that the former problem is generally not determinate whereas the latter one is automatically determinate. This means that, for a given moment sequence, as in (1.2), there will generally be a variety of measures satisfying the same moment conditions (1.2).

Hamburger [Ha] showed that a given sequence $s(0), s(1), \ldots$ has a representation (1.2) for some positive Borel measure $\nu$ on $\mathbb{R}$ if and only if

$$
\sum_{n} \sum_{m} \bar{\xi}_{n} \xi_{m} s(n+m) \geq 0
$$

for all (finite) sequences $\xi_{0}, \xi_{1}, \ldots$. The positive semidefinite condition (5.1) gives rise to a symmetric operator $T$ in a Hilbert space $\mathscr{H}$ where the inner product in $\mathscr{H}$ is defined in terms of the quadratic form (5.1). We shall assume that this symmetric operator, realized on polynomial functions $\sum \xi_{n} x^{n}$ as multiplication by $x$, is essentially selfadjoint, i.e., that it has deficiency-indices $(0,0)$. This assumption has the effect of excluding all the (very interesting) cases [B-C and La] when the problem is indeterminate. (The indeterminate cases are known to admit discrete discontinuous spectrum [B-C].) The following result will be stated for sequences $(s(n))_{0}^{\infty}$ where the above two conditions are satisfied, i.e. (1.2) holds, and the operator $T$ has selfadjoint closure in the corresponding Hilbert space $\mathscr{H}$. Then the spectral resolution $P(\cdot)$ for $\bar{T}$ produces a measure $\nu$ given by

$$
\nu(\cdot)=\langle[1], P(\cdot)[1]\rangle
$$

which is completely analogous to formula (3.4) above, and this measure $\nu$ solves (1.2).

\section{THE SECOND A PRIORI ESTIMATE}

With the above assumptions on the given sequence, the representing measure $\nu$ is unique, and we have

Theorem 6.1. Let $(s(n))_{0}^{\infty}$ be a numerical sequence satisfying the two above mentioned conditions. Then the representing measure $\nu$ is smooth if and only if there is an $\varepsilon \in \mathbb{R}_{+}$with the property that the a priori estimate

$$
\sum_{n} \sum_{m} \bar{\xi}_{n} \xi_{m} s(n+m) \geq \varepsilon\left|\sum(n+1) s(n) \xi_{n+1}\right|^{2}
$$

is satisfied for all complex (finite) sequences $\xi_{0}, \xi_{1}, \xi_{2}, \ldots$.

Proof (Sketch). We shall limit the present discussion since the argument is similar to that of the proof of Theorem 2.1 above. We shall only prove that the condition is sufficient. The necessity is relatively trivial.

By the argument from $\S 3$, we establish a parallel to Lemmas 3.1 and 3.2. For polynomials $p(x)=\sum \xi_{n} x^{n}$, define $p^{\prime}(x)=\sum n \xi_{n} x^{n-1}$, and show the existence of a $\psi \in L^{2}(\nu)$ such that the operator $D$, given by

$$
D p=i p^{\prime}+p \psi
$$

is symmetric in $L^{2}(\nu)$, and moreover satisfies the Heisenberg commutation relation

$$
[D, T]=i I
$$


where $I$ denotes the identity operator in $L^{2}(\nu)$. The proof is based on an extension of (6.2) to a certain enlarged Hilbert space.

Using the argument from $\S 4$, we may then identify the operator system $T, D$ as a compression of a Schrödinger representation [Jo2] acting now on the concrete Hilbert space of vector valued functions $L^{2}(\mathbb{R}, \mathscr{M})$ for some Hilbert space $\mathscr{M}$, accounting for the multiplicity of this Schrödinger representation. If $W: \mathscr{H} \rightarrow L^{2}(\mathbb{R}, \mathscr{M})$ is the isometry which "includes" (see [Jo2, Chapter 4] for details) the $T, D$-system in the Schrödinger representation, then $w:=W[1]$ satisfies

$$
\nu=\|w(x)\|_{\mathscr{M}}^{2} d x
$$

and $w$ is in the domain of the operator $D$. As in $\S 4$ above, this allows us to verify the asserted smoothness property of the representing measure $\nu$.

\section{ACKNOWLEDGMENTS}

The author is pleased to thank Professor R. F. Werner for sending us a pre-publication copy of the paper [We], and we also wish to thank Professor R. T. Powers for several stimulating discussions on commutation relations for operators.

\section{ADDED IN PROOF}

While the conclusions in Theorems 2.1 and 6.1 are given in terms of respective spectral measures from (1.1) and (1.2), it should be stressed that our proof yields additional information, viz., the operator valued measure $F$ from (4.2). While the solutions $F$ in (4.2) are far from unique, the following additional conditions on some solution $F_{0}$ can be shown to imply uniqueness, and as a result we get a new integer valued invariant (alias index) for the two classical moment problems.

Corollary. A solution $F_{0}$ to (4.2) exists as specified such that further (4.4) holds, and $S_{t}:=\int e^{i t \lambda} F_{0}(d \lambda), t \in \mathbb{R}_{+}$, defines a contraction semigroup. When the Stone-von Neumann uniqueness theorem is applied to the minimal unitary dilation (see [J-Mu]) for the system $\left(V, S_{t}\right)$, it follows that the multiplicity of the resulting Weyl operator system is an invariant for the original moment problem.

A similar invariant is also available for the Hamburger moment problem treated in Sections 5 and 6 above. The multiplicity is then defined directly in terms of the Heisenberg group and the corresponding unitary representation which results from consideration of the analogous minimal unitary dilation.

More details for this corollary will appear elsewhere. The author is grateful to M. Putinar and R. F. Werner for their interest and helpful correspondence.

\section{REFERENCES}

[Ak] N. I. Akhiezer, The classical moment problem, Oliver and Boyd, Edinburgh and London, 1965.

[A-G] N. I. Akhiezer and I. M. Glazman, The theory of linear operators in Hilbert space, vol. II, (2nd ed.), Ungar, New York, 1966.

[B-C] Ch. Berg and J. P. R. Christensen, Density questions in the classical theory of moments, Ann. Inst. Fourier (Grenoble) 31 (1981), 99-114. 
[B-R] O. Bratteli and D. W. Robinson, Operator algebras and quantum statistical mechanics, vol. I (2nd ed.), Springer-Verlag, New York, 1987.

[D-S] N. Dunford and J. T. Schwartz, Linear operators, Part I, Interscience, New York, 1967.

[Ha] H. Hamburger, Hermitian transformations of deficiency matrix (1.1), Jacobi matrices, and undetermined moment problems, Amer. J. Math. 66 (1944), 489-522.

[Jo1] P. E. T. Jorgensen, A uniqueness theorem for the Heisenberg-Weyl commutation relations with non-selfadjoint position operator, Amer. J. Math. 103 (1980), 273-287.

[Jo2] _ Operators and representation theory, North-Holland, Amsterdam, 1988.

[J-Mo] P. E. T. Jorgensen and R. T. Moore, Operator commutation relations, Reidel, Dordrecht and Boston, Mass., 1984.

[J-Mu] P. E. T. Jorgensen and P. S. Muhly, Self-adjoint extensions satisfying the Weyl operator commutation relations, J. Analyse Math. 37 (1980), 46-99.

[J-Po] P. E. T. Jorgensen and R. T. Powers, Positive elements for the quantum problem of moments, Preprint, 1989.

[La1] H. J. Landau (ed.), Moments in mathematics, Proc. Sympos. Appl. Math., vol. 37, Amer. Math. Soc., Providence, R.I., 1987.

[La2] __ Maximum entropy and the moment problem, Bull. Amer. Math. Soc. (N.S.) 16 (1987), 47-77.

[Po] R. T. Powers, Selfadjoint algebras of unbounded operators. II, Trans. Amer. Math. Soc. 187 (1974), 261-293.

[We] R. F. Werner, Dilations of symmetric operators shifted by a unitary group, Preprint 1989, J. Funct. Anal. 92 (1990), 166-176.

Department of Mathematics, University of Iowa, Iowa City, Iowa 52242 